\title{
Morphology and isothermal crystallization of graphene oxide reinforced
} biodegradable poly(butylene succinate)

\author{
An-Ke Du ${ }^{1}$, Heng $\mathrm{Li}^{3}$, Ming $\mathrm{Wang}^{2}$, Jian-Bing Zeng ${ }^{2}$, Yi-Dong $\mathrm{Li}^{2} *$ \\ ${ }^{1}$ Chongqing Academy of Science and Technology, Chongqing 401123, China \\ ${ }^{2}$ School of Chemistry and Chemical Engineering, Southwest University, Chongqing \\ 400715, China \\ ${ }^{3}$ Institute of Forensic Science, Criminal Police Corps of Chongqing Public Security \\ Bureau, Chongqing 400021, China
}

Abstract: Graphene oxide (GO) was incorporated into poly(butylene succinate) (PBS) via a solution coagulation method to fabricate PBS/GO nanocomposites. Scanning electron microscope and transmission electron microscope observations indicated that GO with very thin layer dispersed in PBS uniformly and showed a good interfacial adhesion with PBS matrix. Differential scanning calorimetry analysis suggested that the crystallization ability of PBS increased first and then decreased with increase in the content of PBS. Isothermal crystallization kinetics investigation manifested that the overall crystallization rate of PBS increased first and then decreased with increasing PBS content while the crystallization mechanism remained unchanged. Polarized optical microscopy analysis indicated that GO worked as an effective nucleating agent for PBS. X-ray diffraction characterization suggested that incorporation of GO did not change the crystal structure of PBS. Both tensile testing and dynamic mechanical analysis witnessed the reinforcement in mechanical performance of PBS by incorporation of GO. 
Keywords: Poly(butylene succinate); Graphene oxide; Crystallization behavior; Reinforcement

\section{Introduction}

Biobased and biodegradable polymers have attracted ever increasingly attentions due to the increased concerns on environmental problems and resource shortage with respect to traditional petroleum-based non-degradable polymers ${ }^{1-3}$. Poly(butylene succinate) (PBS) is a biodegradable polymer with many attractive properties such as biodegradability, easy processability, thermal stability, and chemical resistance ${ }^{4}$. Furthermore, the main feedstock, succinic acid, has been successfully prepared by a biomass-derived route ${ }^{5}$, which would further promote the development of PBS industry $^{6-8}$. However, PBS still has some disadvantages. For example, it lacks mechanical strength and shows relatively slow crystallization rate, which would restrict the practical application of $\mathrm{PBS}^{8-10}$.

Therefore, many techniques, including chemical copolymerization, polymer blending, and compounding with different fillers, have been employed to improve various properties of $\mathrm{PBS}^{11-24}$. Among those techniques, incorporation of nanofillers to form nanocomposites represents one of the pretty efficient ways to reinforce mechanical properties and enhance crystallization rate of PBS. Thus, various nanofillers such as layered silicate ${ }^{15,25,26}$, carbon nanotubes ${ }^{27-30}$, inorganic nanoparticles ${ }^{31-34}$, cellulose derivatives ${ }^{35-37}$, have been incorporated into PBS for improving physical properties. Graphene and graphene oxide (GO) show many 
supreme properties such as high mechanical strength, Young's modulus, thermal conductivity, gas barrier property, and etc., which makes them ideal reinforcing fillers for various polymer matrices ${ }^{38-40}$. Both graphene and GO have been incorporated into PBS to reinforce mechanical properties ${ }^{9,10,41-44}$. In-situ polymerization and solution processing are the two commonly used methods to incorporate graphene or graphene oxide into PBS.

The dispersion state of nanofillers plays an important role in the final properties of the formed polymer nanocomposites ${ }^{45}$. Graphene or graphene oxide tends to aggregate due to the strong $\pi-\pi$ interaction between layers of graphene or graphene oxide, which usually makes exfoliation difficult and thus leads to poor dispersion in polymer matrix ${ }^{40}$. Exfoliation of graphene in PBS has been achieved by Wang et al. ${ }^{9}$ when preparing PBS/graphene nanocomposite by solution processing. While in the case of PBS/GO composites, solution processing did not achieve exfoliation of GO as reported by Du et $\mathrm{al}^{43,44}$, and the exfoliation of GO was only achieved by in-situ polymerization as reported by Wang and coworkers ${ }^{10}$. While in-situ polymerization seems too complex to practical application, because this method usually requires suitable modification of conventional equipment and polymerization process. It would be better if exfoliated GO can be achieved to disperse in PBS through direct solution processing, because both PBS and GO are commercially available. The loading of exfoliated GO could decrease to show similar crystallization and mechanical behaviors in comparison with platelet-like dispersion. Although the morphology and non-isothermal crystallization kinetics of aggregated GO platelets reinforced PBS 
composites have been reported systematically ${ }^{43,44}$, those of exfoliated GO lamellas reinforced PBS nanocomposites have not been reported. Different crystallization and mechanical behaviors are anticipated if dispersion of GO enhanced.

In this study, we report a way of dispersing largely exfoliated GO into PBS through a solution coagulation method. The morphologies of PBS/GO nanocomposites were studied by scanning electron microscope and transmission electron microscope. The effect of exfoliated GO lamellas on the isothermal crystallization kinetics, spherulitic morphology, crystal structures and mechanical properties of PBS have been studied in detail.

\section{Experimental section}

\subsection{Materials}

Commercial poly(butylene succinate) (PBS) with molecular weight of $1.8 \times 10^{5} \mathrm{~g} / \mathrm{mol}$ was procured from Anqing Hexing Chemical Co., LTD (Anhui, China). Natural graphite flakes were obtained from Nanyang Boxing Mining LTD. Graphene oxide was prepared by oxidation of graphite flakes with a mixture of $\mathrm{H}_{2} \mathrm{SO}_{4}(98 \%)$ and $\mathrm{KMnO}_{4}$ according to the Hummers method. N,N-dimethyl formamide (DMF) and other reagents were obtained from Kelong Chemical Co., LTD (Chengdu, China) and were used as received.

\subsection{Preparation of GO filled PBS nanocomposites}

PBS/GO nanocomposites with GO content from $0.05 \mathrm{wt} \%$ to $1.0 \mathrm{wt} \%$ were prepared though a solution coagulation method. GO was dissolved in deionized water through 
bath ultrasonication to prepare $1.0 \mathrm{wt} \%$ solution. PBS was dissolved in DMF at $80{ }^{\circ} \mathrm{C}$ for $1 \mathrm{hr}$ to prepare a solution with concentration of $5 \%(\mathrm{w} / \mathrm{v})$. Then, a certain amount of GO solution was dropped into predetermined of PBS solution to prepare homogeneous solution, which was then dropped into excessive deionized water to precipitate the PBS/GO nanocomposites. The products were collected by filter and vacuum dried at $60{ }^{\circ} \mathrm{C}$ for $24 \mathrm{hr}$. Five samples with $\mathrm{GO}$ content of $0.05,0.1,0.3,0.5$ and $1.0 \mathrm{wt} \%$ were prepared and for brevity they were named after PBS/GO-0.05, PBS/GO-0.1, PBS/GO-0.3, PBS/GO-0.5, and PBS/GO-1.0, respectively. For property comparison, neat PBS was also treated with the same procedures. For further characterization, the composites were pressed into sheet samples with thickness of 0.5 $\mathrm{mm}$ at $140{ }^{\circ} \mathrm{C}$ under $10 \mathrm{MPa}$.

\subsection{Characterization}

The morphologies of PBS/GO nanocomposites were characterized by scanning electron microscope (SEM) and transmission electron microscope (TEM). The Cryo-fractured surfaces were used for SEM observation. The surfaces were previously sputtered with a layer of gold and then observed by a XL-30s FEG (Philips, Holland)

SEM at an accelerating voltage of $5 \mathrm{kV}$. For TEM observation, the ultrathin sections of ca. $70-80 \mathrm{~nm}$ in thickness were sliced with a Leica EM FC6 cryo-ultramicrotome. The experiment was performed on a JEM-2100F TEM at an accelerating voltage of $200 \mathrm{kV}$.

The thermal properties of PBS and the nanocomposites were characterized by a NETZSCH differential scanning calorimeter (DSC-214). The sample of $\sim 6 \mathrm{mg}$ in 
aluminum pan was first melted at $140{ }^{\circ} \mathrm{C}$ for $3 \mathrm{~min}$ to eliminate any thermal history, then cooled to $-60{ }^{\circ} \mathrm{C}$ at a cooling rate of $10{ }^{\circ} \mathrm{C} / \mathrm{min}$, and finally reheated to $140{ }^{\circ} \mathrm{C}$ at the same rate. All operations were carried out under $\mathrm{N}_{2}$ atmosphere. The cooling and the second heating scans were recorded for data analysis.

Isothermal crystallization kinetics was carried out on the NETZSCH DSC-214 equipment. The sample with $\sim 6 \mathrm{mg}$ was first melted at $140{ }^{\circ} \mathrm{C}$ for $3 \mathrm{~min}$ to remove thermal history, and then quickly cooled to predetermined crystallization temperatures $\left(74,77,80,83\right.$, and $\left.86{ }^{\circ} \mathrm{C}\right)$ at a cooling rate of $60{ }^{\circ} \mathrm{C} / \mathrm{min}$, and finally kept at the temperature until crystallization finished. All the operations were performed under $\mathrm{N}_{2}$ atmosphere. The exothermic curves for isothermal crystallization were recorded for analysis.

Spherulitic morphologies of the samples were observed on an Olympus BX51 polarized optical microscope (POM) with an HT600 hot stage. The sample film within two microscopic cover glasses was first melted at $140{ }^{\circ} \mathrm{C}$ for $3 \mathrm{~min}$ to remove thermal history, and then quickly cooled to $80{ }^{\circ} \mathrm{C}$ at a cooling rate of $60{ }^{\circ} \mathrm{C} / \mathrm{min}$ and maintained at this temperature until crystallization completed. The images were taken for analysis.

X-ray diffraction (XRD) patterns of PBS and the nanocomposites were recorded on a Philips X'Pert X-ray diffractometer with $\mathrm{Cu} \mathrm{K} \alpha$ radiation. The scanning from $5^{\circ}$ to $40^{\circ}$ was carried out at a rate of $2 \%$ min under room temperature.

The mechanical properties were measured on a Sansi Universal Testing Machine (CMT6503) at a crosshead speed of $50 \mathrm{~mm} / \mathrm{min}$ at room temperature. An international 
standard (ISO 527-3-1995) has been referenced during testing. Dumbbell-shaped specimens with width and thickness of 4 and $0.5 \mathrm{~mm}$ were used for the test. The length between the two mechanical grips of the testing machine was $25 \mathrm{~mm}$. Averaged result from five measurements was reported of each sample.

Dynamic mechanical properties of neat PBS and PBS/GO nanocomposites were measured on a TA DMA Q800 instrument under a tensile mode. Tests were performed from $-70{ }^{\circ} \mathrm{C}$ to $60{ }^{\circ} \mathrm{C}$ at a heating rate of $3{ }^{\circ} \mathrm{C} / \mathrm{min}$ and an oscillation frequency of 1 Hz.

\section{Results and discussion}

\subsection{Morphology of PBS/GO nanocomposites}

The final properties of polymer nanocomposites depend strongly on the dispersion of nanofillers and the interfacial adhesion between nanofillers and polymer matrix. It is necessary to characterize the morphologies of $\mathrm{PBS} / \mathrm{GO}$ nanocomposites prepared by the solution coagulation method. SEM is a useful technique to observe the morphology of composite materials. Figure 1 shows the SEM images for the cryo-fractured surfaces of neat PBS and the nanocomposites. Neat PBS showed a homogeneous and smooth surface. Incorporation of GO made the surfaces much rougher, and GO (indicated with red arrow) was found embedded tightly into PBS matrix, manifesting a good interfacial adhesion between PBS and the filled graphene oxide, which was ascribed to the strong interactions between the polar ester groups of PBS and the abundant hydroxyl, carboxyl, and epoxy groups of GO. With increase in 
the content of GO, more GO can be observed on the surface, however, no obvious aggregation could be observed, indicating that solution coagulation is an efficient way to well disperse GO in PBS matrix, which was also approved to be efficient in preparation of some other polymer nanocomposites ${ }^{30,45}$

In order to directly observe the morphologies of the PBS/GO nanocomposites, we performed TEM experiment for the samples. Figure 2 shows the TEM images of the nanocomposites with different compositions. It is seen that exfoliated GO lamellas with wrinkled appearance dispersed in PBS matrix uniformly with the content of GO less than $0.5 \mathrm{wt} \%$. When the content of GO increased up to more than $0.5 \mathrm{wt} \%$, exfoliated GO lamellas were also observed, meanwhile, some GO aggregates could be observed from TEM images of PBS/GO-0.5 (Figure 2d) and PBS/GO-1.0 (Figure 2e and $\mathrm{f}$ ). The aggregation was attributed to the increased volume fraction of well dispersed GO lamella with increasing its content. The exfoliation of GO in the present PBS/GO nanocomposites was attributed to the use of ultrasound during dispersion of GO in water and the coagulation technique. Ultrasound facilitates exfoliation of GO in aqueous medium and coagulation process prevents aggregation of exfoliated GO lamellas by surface wrapping of PBS. We noticed that ultrasound was not applied in preparation of $\mathrm{PBS} / \mathrm{GO}$ nanocomposites in previously reported literatures, where the technique to remove solvent was evaporation ${ }^{43,44}$. Simple stir may not provide sufficient force to exfoliate GO and they may tend to aggregate during long-time evaporation process, which may account for the platelet-like dispersion of GO within the prepared PBS/GO composites. 


\subsection{Thermal behavior of PBS/GO nanocomposites}

The effect of GO on the thermal properties including glass transition, crystallization and melting parameters of PBS was studied by differential scanning calorimetry. Figure 3 shows the DSC cooling and the seconding heating scans of neat PBS and the nanocomposites, and Table 1 summarizes the corresponding parameters including glass transition temperature $\left(T_{\mathrm{g}}\right)$, crystallization temperature $\left(T_{\mathrm{c}}\right)$, crystallization enthalpy $\left(\Delta H_{\mathrm{c}}\right)$, melting temperature $\left(T_{\mathrm{m}}\right)$, and fusion enthalpy $\left(\Delta H_{\mathrm{m}}\right)$. The $T_{\mathrm{g}}$ of PBS showed uptrend with increase in the content of GO, which is reasonable because the good interfacial interaction and lamella structure of GO inevitably reduce the chain mobility of PBS. The content of GO played an important role in the crystallization of the composites. Neat PBS showed a wide crystallization peak with a $T_{\mathrm{c}}$ of $60.6{ }^{\circ} \mathrm{C}$. The crystallization peak narrowed, the $T_{\mathrm{c}}$ and $\Delta H_{\mathrm{c}}$ increased by incorporation of GO, indicating enhanced crystallization. It is interesting to find that the $T_{\mathrm{c}}$ increased first and then decreased with increase in the content of GO, which means that the crystallizability of PBS in the composites increased first and then decreased. The presence of lamellar GO could not only provide nucleating sites but also form confined spaces for crystallization of PBS. The nucleating effect is helpful for accelerating crystallization rate while confined spaces would restrict chain mobility of PBS to reduce crystallization rate. The reduction in crystallization rate was also observed in graphene oxide filled poly(lactic acid) composites, where the crystallization of poly(lactic acid) occurred in confined spaces formed by graphene 
oxide nanosheets ${ }^{46}$. The best balance of the two effects occurred when the content of GO was $0.05 \mathrm{wt} \%$ in the present study, which is different from the result reported by Du and coworkers ${ }^{43}$. In that study, $0.5 \mathrm{wt} \%$ was the optimal content for enhancing crystallization of PBS. The difference was possibly attributed to the different dispersion state of GO in the composites. The GO existed with platelets consist of multiple layers in their study while the GO in the present study was almost exfoliated with very thin lamellas dispersed uniformly in PBS matrix. With same content, the exfoliated lamella would take much more volume fraction, which then caused crystallization of PBS in more confined spaces, leading to smaller crystal growth rate. The $T_{\mathrm{m}}$ of PBS increased slightly by incorporation of GO. This can be explained by the fact that crystals formed at higher temperature showed better thermal stability. The incorporation of GO shifted the crystallization to higher temperatures, thus the crystals of PBS formed within PBS/GO nanocomposites were more stable than those in neat PBS. In addition, the $\Delta H_{\mathrm{m}}$ of PBS/GO nanocomposites also increased slightly compared to neat PBS due to the enhanced crystallization.

\subsection{Isothermal crystallization kinetics of PBS/GO nanocomposites}

In order to study the effect of GO on the crystallization of PBS in detail, the isothermal crystallization kinetics of neat PBS and some typical nanocomposites were investigated by DSC in a wide range of crystallization temperatures. Figure 4a shows the plots of relative crystallinity $\left(X_{\mathrm{t}}\right)$ versus crystallization time at various crystallization temperatures for neat PBS. All plots showed similar sigmoid shapes 
and the time for PBS to complete crystallization increased with increasing crystallization temperature, indicating crystallization rate decreased in the temperature range considered in this study. For comparison, Figure $4 \mathrm{~b}$ shows the plots of relative crystallinity $\left(X_{\mathrm{t}}\right)$ versus crystallization time for neat PBS and the nanocomposites at $80{ }^{\circ} \mathrm{C}$. It is obvious that the time required for finishing crystallization decreased first and then increased with increasing content of GO, which is in agreement with tendency obtained by non-isothermal crystallization as discussed above. The plots at other temperatures showed similar curves, and for brevity, were not shown here.

The well-known Avrami equation was used to describe the isothermal crystallization kinetics of polymers. The equation assumes that the relationship of relative crystallinity $\left(X_{\mathrm{t}}\right)$ and crystallization time $(t)$ follows

$$
1-X_{t}=\exp \left(-\mathrm{k} t^{n}\right)
$$

where $X_{\mathrm{t}}$ is relative crystallinity at a given time $\mathrm{t}, \mathrm{k}$ is a rate constant, and $\mathrm{n}$ is the Avrami exponent, which denotes the nature of the nucleation and growth process ${ }^{47}$. Double logarithm changes the Avrami equation to

$$
\log \left(-\ln \left(1-X_{t}\right)=\log k+n \log t\right.
$$

The Avrami equation is applicable if a straight line is obtained from plotting $\log \left(-\ln \left(1-X_{\mathrm{t}}\right)\right.$ with $\log t$. The rate constant and the Avrami exponent can be deduced from the intercept and slope of plotting straight line. Figure 5a shows the Avrami plots of neat PBS at different temperatures. Almost five parallel straight lines were obtained, indicating that the Avrami equation is suitable to describe the isothermal crystallization kinetics of PBS. Figure 5b also shows a series of straight lines for the 
Avrami plots of PBS/GO nanocomposites, which indicate the feasibility of using the Avrami equation to analyze the isothermal crystallization kinetics of the composites. Accordingly, the values of $k$ and $n$ at different temperatures for neat PBS and the typical nanocomposites can be calculated, and the results are summarized in Table 2 . It is found that the $\mathrm{n}$ values at different temperatures for all samples were in the range of 2.1-2.5, which indicate that the incorporation of GO did not change the crystallization mechanism of PBS and they all followed a mechanism of three dimensional truncated spherulitic growth with athermal nucleation ${ }^{48,49}$.

The $k$ values were various for different sample at a given temperature, however, it is unsuitable to compare the crystallization rate directly from the $k$ values because the $n$ values are also variable. Another parameter crystallization half-life time $\left(t_{0.5}\right)$, the time required to achieve relative crystallinity of $50 \%$, is useful to describe isothermal crystallization kinetics. The reciprocal of $t_{0.5}$, i.e., $1 / t_{0.5}$, can be used directly to express the overall crystallization rate. The value of $t_{0.5}$ is calculated by

$$
t_{0.5}=(\ln 2 / k)^{1 / n}
$$

Then, $1 / t_{0.5}$ can be obtained from the values of $k$ and $n$, and the results are also shown in Table 2. It is obvious that the values of $1 / t_{0.5}$ decreased with increasing crystallization temperature for the given sample, indicating a slower overall crystallization rate at a higher temperature considered in this study. What we focus more is the effect of GO on the crystallization rate of the composites. The overall crystallization rate of PBS increased first and then decreased with increasing GO content from 0 to $0.5 \mathrm{wt} \%$, the maximum overall crystallization rate occurred at GO 
content of $0.05 \mathrm{wt} \%$ for the give temperatures. As above section mentioned, GO played two opposite effects on the crystallization of PBS, the optimal balance between positive nucleation effect and negative crystal growth rate restriction effect was achieved when $0.05 \mathrm{wt} \% \mathrm{GO}$ was incorporated.

In order to observe the nucleation effect of GO for isothermal crystallization of PBS, the spherulitic morphologies of neat PBS and the nanocomposites were observed by POM. Figure 6 shows the POM images of the samples after isothermally crystallized at $80{ }^{\circ} \mathrm{C}$. Neat PBS showed well developed large spherulites with clear boundaries. The nucleation effect of GO was remarkable, as evidenced by the decreased size of spherulites and increased number of spherulites with incorporation and increasing content of GO. The spherulitic boundaries were even hard to distinguish when the content of GO increased to $1.0 \mathrm{wt} \%$.

\subsection{X-ray diffraction analysis}

XRD was carried out to study the effect of GO on the crystal structure of PBS. Figure 7 shows the XRD patterns of neat PBS and PBS/GO nanocomposites. Neat PBS has three main characteristic diffraction peaks at $2 \theta$ of 19.7, 21.9, and $22.6^{\circ}$, corresponding to the (020), (021), and (110) planes of PBS, respectively ${ }^{8,50}$. The positions of the three diffraction peaks remained unchanged after incorporation of GO with various contents, which indicated that incorporation of GO into PBS to form nanocomposites does not change the crystal structure of PBS. It is reported that characteristic peak of interlayer spacing of GO locates at $2 \theta$ of less than $10^{\circ}$. But it 
could not be observed on the curves of all the composites. Although this does not necessarily indicate completely exfoliation, it does manifest the disordered stacking structure of the graphene oxide ${ }^{9,51}$.

\subsection{Mechanical properties}

Graphene oxide filled polymer composites usually showed reinforced mechanical performance. The static mechanical properties of PBS and PBS/GO nanocomposites were characterized by tensile test to study the effect of GO on the tensile strength, Young's modulus, and elongation at break of PBS. Figure 8 shows the stress-strain curves of neat PBS and PBS/GO nanocomposites with different GO loadings. Neat PBS showed an obvious yielding point at the stress of $\sim 22.6 \mathrm{MPa}$. Strain hardening occurred for neat PBS before breaking, which resulted in a higher tensile strength than yielding strength. The elongation at break, tensile strength and Young's modulus of neat PBS was $\sim 305 \%, 25.3 \mathrm{MPa}$ and $321.8 \mathrm{MPa}$, respectively, as shown in Table 3. PBS/GO nanocomposites also showed distinct yielding points with GO content of less than $1.0 \mathrm{wt} \%$. When the content of GO increased to $1.0 \mathrm{wt} \%$, the nanocomposites broke before yielding, indicating behavior of brittle fracture. No strain hardening occurred for all the PBS/GO nanocomposites. The yielding strength equaled to tensile strength of PBS/GO nanocomposites. It is interesting to find that the yielding strength of PBS was increased obviously with incorporation of GO and increasing its content, indicating effective reinforcement of the composites. The evidence of reinforcement could also be proved from the change of Young's modulus of the composites versus 
the content of GO. Young's modulus was obtained from the slope of the linear range of the stress-strain curve. The inset of Figure 8 showed that the slope increased gradually with increasing GO content, indicating an increase in Young's modulus with GO content. The values are summarized in Table 3. The elongation at break decreased gradually with increasing GO content. The result is similar to other nanofillers reinforced polymer composites, which is reasonable because reinforcement in mechanical strength usually accompanied by the loss in ductility of polymer matrix.

Except for static mechanical properties, dynamic mechanical properties of neat PBS and PBS/GO nanocomposites were also investigated to reinforcement of GO on the modulus of the PBS. Figure 9 shows the dynamic storage modulus as a function of temperature for neat PBS and PBS/GO composites. Storage modulus showed drastic drop at around $-30{ }^{\circ} \mathrm{C}$, corresponding to $\alpha$-relaxation (i.e., glass transition temperature) of PBS. It is noted that the storage modulus increased with increasing content of GO in the full temperature range considered in this study, indicating reinforced mechanical performance. Especially, storage modulus below $\alpha$-relaxation increased drastically with increase in the content of GO. For example, the storage modulus of neat PBS at $-60{ }^{\circ} \mathrm{C}$ was $3229 \mathrm{MPa}$. The values were 4973, 5514, 6227,7274, and 8811 MPa for PBS/GO-0.05, PBS/GO-0.1, PBS/GO-0.3, PBS/GO-0.5, and PBS/GO-1.0, respectively.

\section{Conclusions}

Graphene oxide was successfully incorporated into PBS to form PBS/GO 
nanocomposites via a solution coagulation method. Graphene oxide was largely exfoliated and dispersed uniformly in PBS matrix and showed good interfacial adhesion with PBS. The content of GO played an important role in crystallization of PBS. The crystallization temperature increased first and then decreased with increase in the content of GO due to the two competitive effects of GO on the crystallization of PBS. The well dispersed GO lamellas worked as nucleating sites and confined space for crystallization of PBS. The former is positive while the latter is negative for crystallization of PBS. The best balance was observed for the composites containing $0.05 \mathrm{wt} \% \mathrm{GO}$. Isothermal crystallization kinetics analysis indicated the overall crystallization rate of PBS increased first and then decreased with increase in the content of GO, while the crystallization mechanism remained unchanged. Incorporation of GO resulted in smaller spherulite due to the increased nucleating density but did not change the crystal structure of PBS. The mechanical properties including yielding strength, tensile strength, Young's modulus and storage modulus of PBS were obviously reinforced by incorporation of GO, meanwhile, the elongation at break decreased.

\section{Acknowledgements}

This work was supported by National Science Foundation of China (51373107) and Fundamental Research Funds for the Central Universities (SWU115006).

\section{References}


(1) M. M. Reddy, S. Vivekanandhan, M. Misra, S. K. Bhatia, A. K. Mohanty, Biobased plastics and bionanocomposites: Current status and future opportunities, Prog. Polym. Sci. 38 (2013) 1653-1689.

(2) A. Gandini, T. M. Lacerda, From monomers to polymers from renewable resources: Recent advances, Prog. Polym. Sci. 48 (2015) 1-39.

(3) T. H. Zhao, Y. He, Y. D. Li, M. Wang, J. B. Zeng, Dynamic vulcanization of castor oil in a polylactide matrix for toughening, RSC Adv. 6 (2016) $79542-79553$.

(4) J. Xu, B. H. Guo, Poly(butylene succinate) and its copolymers: Research, development and industrialization, Biotechnol. J. 5 (2010) 1149-1163.

(5) J. G. Zeikus, M. K. Jain, P. Elankovan, Biochemical basis for carbon monoxide tolerance and butanol production by butyribacterium methylotrophicum, Appl. Microbiol. Biotechnol. 51 (1999) 545-552.

(6) J. B. Zeng, F. Wu, C. L. Huang, Y. S. He, Y. Z. Wang, Urethane Ionic Groups Induced Rapid Crystallization of Biodegradable Poly(ethylene succinate), ACS Macro Lett. 1 (2012) 965-968.

(7) L. Lv, F. Wu, S. C. Chen, Y. Z. Wang, J. B. Zeng, Properties regulation of poly(butylene succinate) ionomers through their ionic group distribution, Polymer 66 (2015) 148-159.

(8) F. Wu, C. L. Huang, J. B. Zeng, S. L. Li, Y. Z. Wang, Synthesis and characterization of segmented poly(butylene succinate) urethane ionenes containing secondary amine cation, Polymer 55 (2014) 4358-4368. 
(9) X. Wang, H. Yang, L. Song, Y. Hu, W. Xing, H. Lu, Morphology, mechanical and thermal properties of graphene-reinforced poly(butylene succinate) nanocomposites, Compos. Sci. Tech.72 (2011) 1-6.

(10)X. W. Wang, C. A. Zhang, P. L. Wang, J. Zhao, W. Zhang, J. H. Ji, K. Hua, J. Zhou, X. B. Yang, X. P. Li, Enhanced Performance of Biodegradable Poly(butylene succinate)/Graphene Oxide Nanocomposites via in Situ Polymerization, Langmuir 28 (2012) 7091-7095.

(11)J. B. Zeng, L. Jiao, Y. D. Li, M. Srinivasan, T. Li, Y. Z. Wang, Bio-based blends of starch and poly(butylene succinate) with improved miscibility, mechanical properties, and reduced water absorption, Carbohydr. Polym. 83 (2011) 762-768.

(12)S. D. Ding, G. C. Zheng, J. B. Zeng, L. Zhang, Y. D. Li, Y. Z. Wang, Preparation, characterization and hydrolytic degradation of poly[p-dioxanone-(butylene succinate)] multiblockcopolymer, Eur. Polym. J. 45 (2009) 3043-3057.

(13) Y. S. He, J. B. Zeng, S. L. Li, Y. Z. Wang, Crystallization behavior of partially miscible biodegradable poly(butylene succinate)/poly(ethylene succinate) blends, Thermochim. Acta 529 (2012) 80-86.

(14)J. B. Zeng, Q. Y. Zhu, X. Lu, Y. S. He, Y. Z. Wang, From miscible to partially miscible biodegradable double crystalline poly (ethylene succinate)-b-poly(butylene succinate) multiblock copolymers, Polym. Chem. 3 (2012) 399-408.

(15)S. S. Ray, K. Okamoto, M. Okamoto, Structure-property relationship in biodegradable poly(butylene succinate)/layered silicate nanocomposites, 
Macromolecules 36 (2003) 2355-2367.

(16)S. Y. Hwang, E. S. Yoo, S. S. Im, The synthesis of copolymers, blends and composites based on poly(butylene succinate), Polym. J. 44 (2012) 1179-1190.

(17)Y. Zhang, C. Yu, P. K. Chu, F. Lv, C. Zhang, J. Ji, R. Zhang, H. Wang, Mechanical and thermal properties of basalt fiber reinforced poly(butylene succinate) composites, Mater. Chem. Phys. 133 (2012) 845-849.

(18) V. Ojijo, S. S. Ray, Processing strategies in bionanocomposites, Prog. Polym. Sci. 38 (2013) 1543-1589.

(19)H. Wang, P. G. Ren, C. Y. Liu, L. Xu, Z. M. Li, Enhanced toughness and strength of conductive cellulose-poly(butylene succinate) films filled with multiwalled carbon nanotubes, Cellulose 21 (2014) 1803-1812.

(20)J. B. Chen, J. Z. Xu, H. Xu, Z. M. Li, G. J. Zhong, J. Lei, The crystallization behavior of biodegradable poly(butylene succinate) in the presence of organically modified clay with a wide range of loadings, Chin. J. Polym. Sci. 33 (2015) $576-586$

(21)Q. Chen, X. Wen, H. Chen, Y. Qi, J. Gong, H. Yang, Y. Li, T. Tang, Study of the effect of nanosized carbon black on flammability and mechanical properties of poly(butylene succinate). Polym. Adv. Tech. 26 (2015) 128-135.

(22)X. Lu, L. Tang, L. Wang, J. Zhao, D. Li, Z. Wu, P. Xiao, Morphology and properties of bio-based poly (lactic acid)/high-density polyethylene blends and their glass fiber reinforced composites, Polym. Test. 54 (2016) 90-97.

(23)L. Sisti, J. Belcari, L. Mazzocchetti, G. Totaro, M. Vannini, L. Giorgini, A. 
Zucchelli, A. Celli, Multicomponent reinforcing system for poly(butylene succinate): Composites containing poly(L-lactide) electrospun mats loaded with graphene, Polym. Test. 50 (2016) 283-291.

(24)S. Y. Zhou, J. B. Chen, X. J. Li, X. Ji, G. J. Zhong, Z. M. Li, Innovative enhancement of gas barrier properties of biodegradable poly(butylene succinate) nanocomposite films by introducing confined crystals, RSC Adv. 6 (2016) $2530-2536$.

(25)S. S. Ray, K. Okamoto, P. Maiti, M. Okamoto, New poly(butylene succinate)/layered silicate nanocomposites: Preparation and mechanical properties, J. Nanosci. Nanotech. 2 (2002) 171-176.

(26)G. X. Chen, J. S. Yoon, Nonisothermal crystallization kinetics of poly(butylene succinate) composites with a twice functionalized organoclay, J. Polym. Sci. Part B-Polym. Phys. 43 (2005) 817-826.

(27)Y. F. Shih, L. S. Chen, R. J. Jeng, Preparation and properties of biodegradable PBS/multi-walled carbon nanotube nanocomposites, Polymer 49 (2008) $4602-4611$.

(28)K. P. Pramoda, N. T. T. Linh, C. Zhang, T. Liu, Multiwalled Carbon Nanotube Nucleated Crystallization Behavior of Biodegradable Poly(butylene succinate) Nanocomposites, J. Appl. Polym. Sci. 111 (2009) 2938-2945.

(29)L. Tan, Y. Chen, W. Zhou, S. Ye, J. Wei, Novel approach toward poly(butylene succinate)/single-walled carbon nanotubes nanocomposites with interfacial-induced crystallization behaviors and mechanical strength, Polymer 52 
(2011) 3587-3596.

(30)R. T. Zeng, W. Hu, M. Wang, S. D. Zhang, J. B. Zeng, Morphology, rheological and crystallization behavior in non-covalently functionalized carbon nanotube reinforced poly(butylene succinate) nanocomposites with low percolation threshold, Polym. Test. 50 (2016) 182-190.

(31)R. Y. Chen, W. Zou, H. C. Zhang, G. Z. Zhang, Z. T. Yang, G. Jin, J. P. Qu, Thermal behavior, dynamic mechanical properties and rheological properties of poly(butylene succinate) composites filled with nanometer calcium carbonate, Polym. Test. 42 (2015) 160-167.

(32)W. G. Liu, X. C. Zhang, H. Y. Li, Z. Liu, Effect of surface modification with 3-aminopropyltriethyloxy silane on mechanical and crystallization performances of $\mathrm{ZnO} /$ poly(butylenesuccinate) composites, Compos. Part B-Eng. 43 (2012) 43, 2209-2216.

(33)C. W. Zhou, J. X. Zhao, Q. Liu, B. Meng, Z. H. Wu, Study on Mechanical, Thermal Properties and Morphology of Biodegradable Poly(butylene succinate)/Nano-TiO 2 Composites, Int. Polym. Proc. 28 (2013) 483-488.

(34)W. Zhou, T. Xu, X. Wang, E. Zhi, J. Liu, W. Zhang, J. Ji, In situ polymerized nanocomposites of poly(butylene succinate)/ $/ \mathrm{TiO}_{2}$ nanofibers: molecular weight, morphology, and thermal properties, J. Appl. Polym. Sci. 2013, 127, 733-739.

(35)F. Hu, N. Lin, P. R. Chang, J. Huang, Reinforcement and nucleation of acetylated cellulose nanocrystals in foamed polyester composites, Carbohydr. Polym. 129 (2015) 208-215. 
(36)M. Zhou, M. Fan, Y. Zhao, T. Jin, Q. Fu, Effect of stretching on the mechanical properties in melt-spun poly(butylene succinate)/microfibrillated cellulose (MFC) nanocomposites. Carbohydr. Polym. 140 (2016) 383-392.

(37)D. M. Panaitescu, A. N. Frone, I. Chiulan, Nanostructured biocomposites from aliphatic polyesters and bacterial cellulose, Ind. Crop. Prod. 93 (2016) 251-266.

(38)K. S. Novoselov, V. I. Fal'ko, L. Colombo, P. R. Gellert, M. G. Schwab, K. Kim, A roadmap for graphene, Nature 490 (2012) 192-200.

(39)X. Huang, X. Qi, F. Boey, H. Zhang, Graphene-based composites. Chem. Soc. Rev. 42 (2012) 666-686.

(40)T. Kuilla, S. Bhadra, D. Yao, N. H. Kim, S. Bose, J. H. Lee, Recent advances in graphene based polymer composites, Prog. Polym. Sci. 35 (2010) 1350-1375.

(41)C. Wan, B. Chen, Reinforcement of biodegradable poly(butylene succinate) with low loadings of graphene oxide, J. Appl. Polym. Sci. 127 (2013) 5094-5099.

(42)T. X. Jin, C. Liu, M. Zhou, S. G. Chai, F. Chen, Q. Fu, Crystallization, mechanical performance and hydrolytic degradation of poly(butylene succinate)/graphene oxide nanocomposites obtained via in situ polymerization, Compos. Part A 68 (2015) 193-201.

(43)X. C. Du, X. L. Xu, X. H. Liu, J. H. Yang, Y. Wang, X. L. Gao, Graphene oxide induced crystallization and hydrolytic degradation of poly(butylene succinate), Polym. Degrad. Stab. 123 (2016) 94-104.

(44)X. C. Du, Y. P. Wang, W. B. Huang, J. H. Yang, Y. Wang, Rheology and non-isothermal crystallization behaviors of poly(butylene succinate)/graphene 
oxide composites, Colloid Polym. Sci. 293 (2015) 389-400.

(45)M. Wang, X. Y. Deng, A. K. Du, T. H. Zhao, J. B. Zeng, Poly(sodium 4-styrenesulfonate) modified graphene for reinforced biodegradable poly(epsilon-caprolactone) nanocomposites, RSC Adv. 5 (2015) 73146-73154.

(46)H. D. Huang, J. Z. Xu, Y. Fan, L. Xu, Z. M. Li, Poly(l-lactic acid) Crystallization in a Confined Space Containing Graphene Oxide Nanosheets. J. Phys. Chem. B 117 (2013) 10641-10651.

(47)M. Avrami, Kinetics of Phase Change. I. General Theory. J. Chem. Phys. 7 (1939) 1103-1112.

(48)J. B. Zeng, Q. Y. Zhu, Y. D. Li, Z. C. Qiu, Y. Z. Wang, Unique Crystalline/Crystalline Polymer Blends of Poly(ethylene succinate) and Poly(p-dioxanone): Miscibility and Crystallization Behaviors, J. Phys. Chem. B 114 (2010) 14827-14833.

(49)X. Lu, J. B. Zeng, C. L. Huang, Y. Z. Wang, Isothermal Crystallization Behavior of Biodegradable P(BS-b-PEGS) Multiblock Copolymers, Ind. Eng. Chem. Res. 51 (2012) 8262-8272.

(50)J. B. Zeng, C. L. Huang, L. Jiao, X. Lu, Y. Z. Wang, X. L. Wang, Synthesis and Properties of Biodegradable Poly(butylene succinate-co-diethylene glycol succinate) Copolymers, Ind. Eng. Chem. Res. 51 (2012) 12258-12265.

(51)H. He, J. Klinowski, M. Forster, A. Lerf, A new structural model for graphite oxide, Chem. Phys. Lett. 287 (1998) 53-56. 


\section{Tables and Figures}

Table 1. Thermal properties of neat PBS and PBS/GO nanocomposites.

\begin{tabular}{llllll}
\hline Sample & $T_{\mathrm{g}}\left({ }^{\circ} \mathrm{C}\right)$ & $T_{\mathrm{c}}\left({ }^{\circ} \mathrm{C}\right)$ & $\Delta H_{\mathrm{c}}(\mathrm{J} / \mathrm{g})$ & $T_{\mathrm{m}}\left({ }^{\circ} \mathrm{C}\right)$ & $\Delta H_{\mathrm{m}}(\mathrm{J} / \mathrm{g})$ \\
\hline PBS & -39.4 & 60.6 & 61.53 & 104.4 & 61.16 \\
PBS-0.05 & -38.6 & 66.1 & 62.23 & 105.0 & 62.61 \\
PBS-0.1 & -37.8 & 63.8 & 62.36 & 104.8 & 63.23 \\
PBS-0.3 & -37.6 & 63.0 & 62.37 & 105.0 & 63.13 \\
PBS-0.5 & -37.2 & 61.4 & 62.24 & 105.3 & 63.19 \\
PBS-1.0 & -35.0 & 61.4 & 61.38 & 105.2 & 62.15 \\
\hline
\end{tabular}


Table 2. Parameters for isothermal crystallization kinetics of neat PBS and PBS/GO nanocomposites

\begin{tabular}{lllll}
\hline Sample & $\begin{array}{l}\text { Temperature } \\
\left({ }^{\circ} \mathrm{C}\right)\end{array}$ & $\mathrm{n}$ & $\begin{array}{l}\mathrm{K} \\
\left(\mathrm{min}^{-\mathrm{n}}\right)\end{array}$ & $\begin{array}{l}1 / t_{0.5} \\
\left(\mathrm{~min}^{-1}\right)\end{array}$ \\
\hline Neat PBS & 74 & 2.27 & 0.30 & 0.69 \\
& 77 & 2.32 & $5.55 \times 10^{-2}$ & 0.34 \\
& 80 & 2.19 & $1.71 \times 10^{-2}$ & 0.18 \\
& 83 & 2.13 & $4.20 \times 10^{-3}$ & 0.09 \\
PBS/GO-0.05 & 86 & 2.32 & $6.66 \times 10^{-4}$ & 0.05 \\
& 74 & 2.15 & 0.44 & 0.81 \\
& 77 & 2.21 & 0.11 & 0.44 \\
& 80 & 2.30 & $7.12 \times 10^{-2}$ & 0.37 \\
& 83 & 2.22 & $4.93 \times 10^{-3}$ & 0.11 \\
& 86 & 2.36 & $5.97 \times 10^{-4}$ & 0.05 \\
PBS/GO-0.1 & 74 & 2.22 & 0.41 & 0.79 \\
& 77 & 2.16 & 0.11 & 0.43 \\
& 80 & 2.35 & $1.38 \times 10^{-2}$ & 0.19 \\
& 83 & 2.40 & $2.71 \times 10^{-3}$ & 0.10 \\
& 86 & 2.41 & $4.32 \times 10^{-4}$ & 0.05 \\
PBS/GO-0.5 & 74 & 2.20 & 0.31 & 0.69 \\
& 77 & 2.13 & $7.12 \times 10^{-2}$ & 0.34 \\
& 80 & 2.27 & $1.11 \times 10^{-2}$ & 0.16 \\
& 83 & 2.20 & $3.24 \times 10^{-3}$ & 0.09 \\
& 86 & 2.12 & $6.42 \times 10^{-4}$ & 0.04 \\
\hline
\end{tabular}


Table 3. Mechanical properties of neat PBS and PBS/GO nanocomposites

\begin{tabular}{llll}
\hline Sample & $\begin{array}{c}\text { Elongation at break } \\
(\%)\end{array}$ & $\begin{array}{c}\text { Tensile strength } \\
(\mathrm{MPa})\end{array}$ & $\begin{array}{c}\text { Young's modulus } \\
(\mathrm{MPa})\end{array}$ \\
\hline PBS & $305 \pm 15$ & $25.3 \pm 0.8$ & $321.8 \pm 7.0$ \\
PBS-0.05 & $270 \pm 12$ & $27.4 \pm 0.5$ & $337.3 \pm 6.6$ \\
PBS-0.1 & $185 \pm 12$ & $27.3 \pm 1.0$ & $342.4 \pm 15.3$ \\
PBS-0.3 & $131 \pm 16$ & $27.7 \pm 0.5$ & $354.7 \pm 13.2$ \\
PBS-0.5 & $32 \pm 7$ & $27.8 \pm 0.6$ & $370.4 \pm 10.4$ \\
PBS-1.0 & $16 \pm 1$ & $26.6 \pm 0.6$ & $397.1 \pm 3.5$ \\
\hline
\end{tabular}



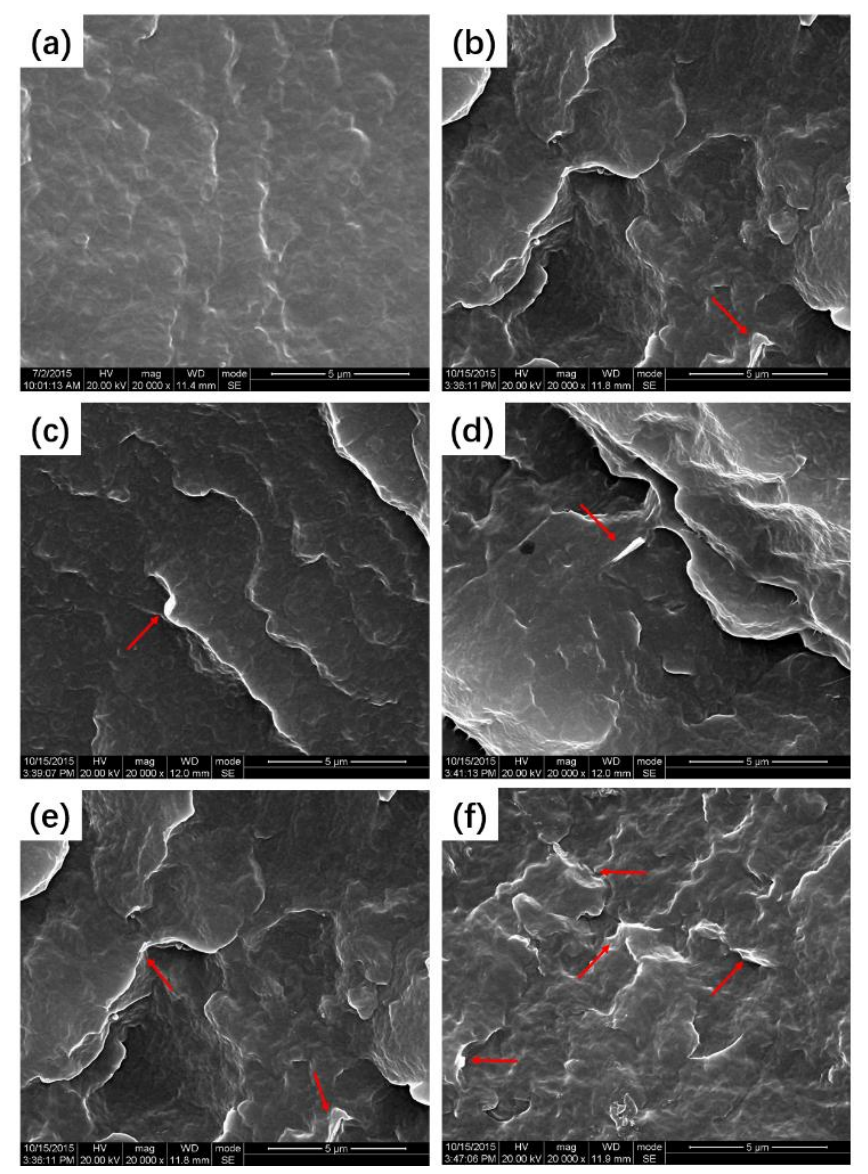

Figure 1. SEM images for the cryo-fractured surfaces of neat PBS (a), PBS/GO-0.05

(b), PBS/GO-0.1 (c), PBS/GO-0.3 (d), PBS/GO-0.5 (e), and PBS/GO-1.0 (f). 

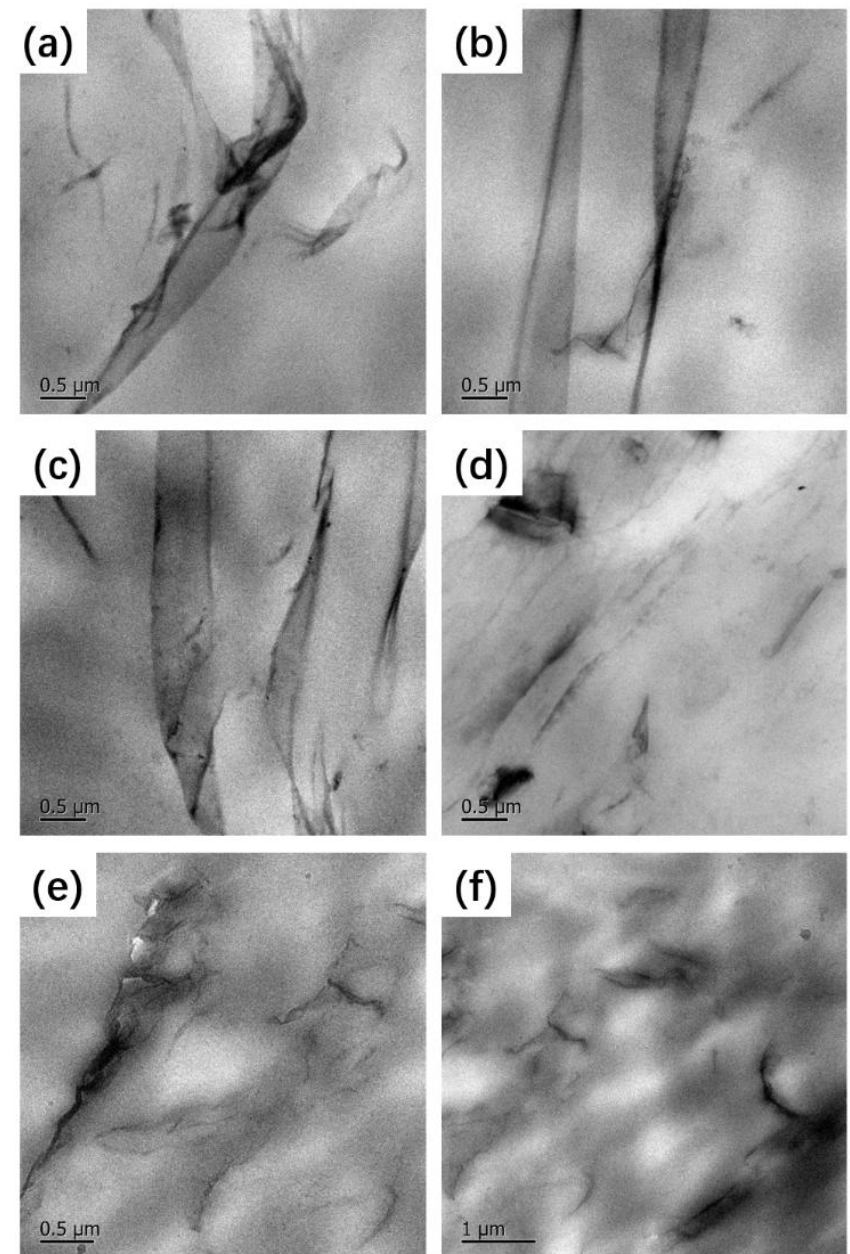

Figure 2. TEM images with high magnification for PBS/GO-0.05 (a), PBS/GO-0.1 (b), PBS/GO-0.3 (c), PBS/GO-0.5 (d), PBS/GO-1.0 (e), and with low magnification of PBS/GO-1.0 (f). 

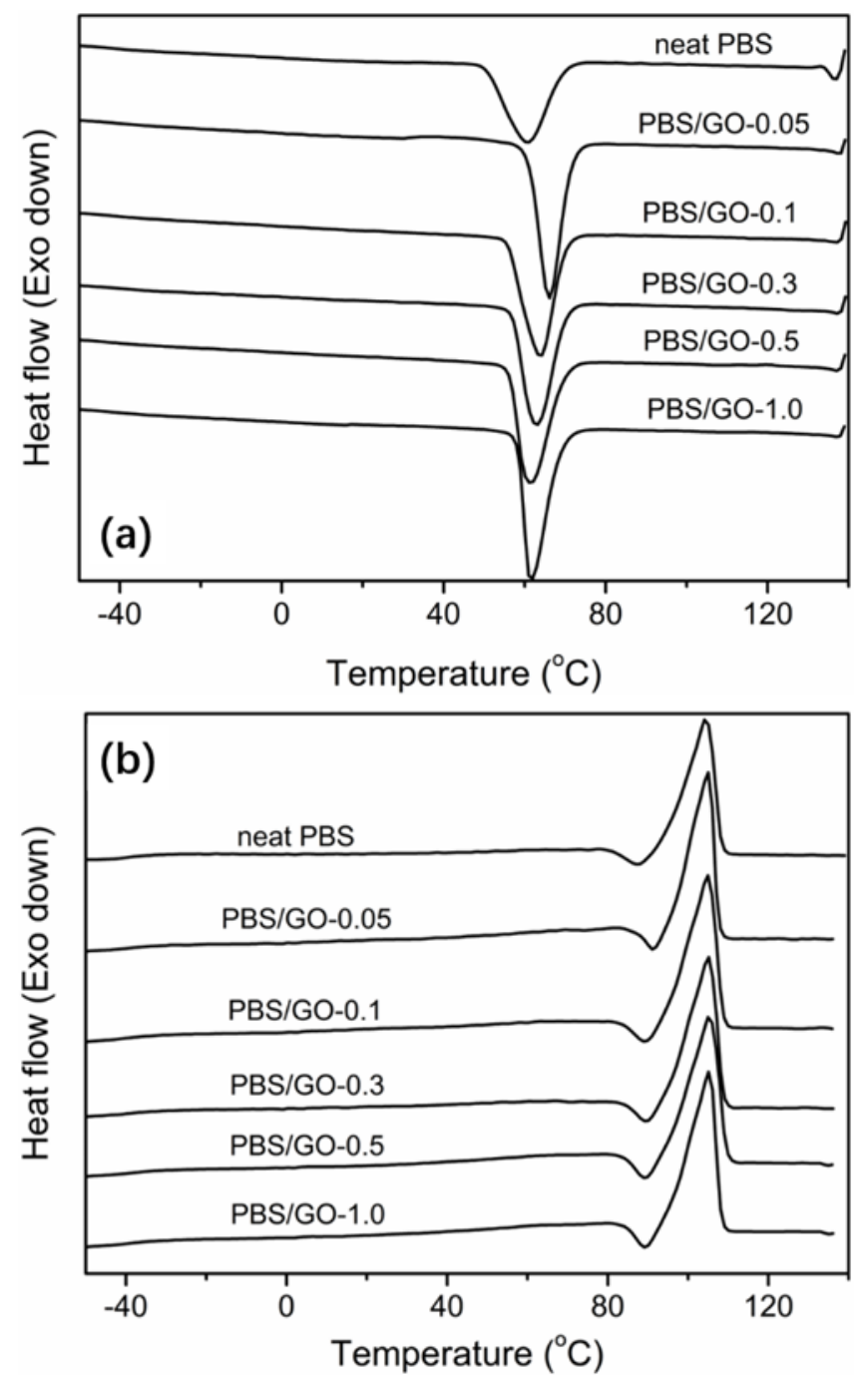

Figure 3. DSC cooling (a) and the second heating scans (b) of neat PBS and PBS/GO nanocomposites. 

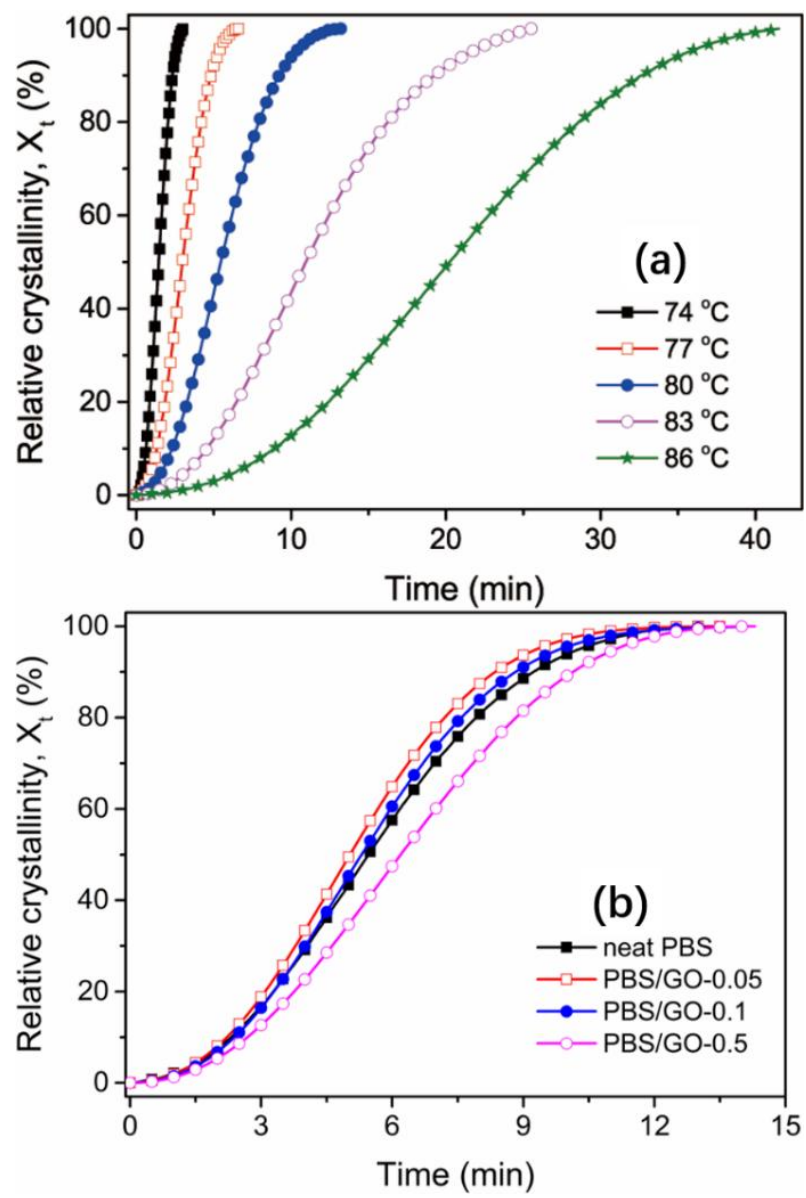

Figure 4. (a) Plots of relative crystallinity $\left(X_{\mathrm{t}}\right)$ versus crystallization time at various crystallization temperatures for neat PBS and (b) plots of relative crystallinity $\left(X_{\mathfrak{t}}\right)$ versus crystallization time at $80{ }^{\circ} \mathrm{C}$ for PBS and PBS/GO nanocomposites. 

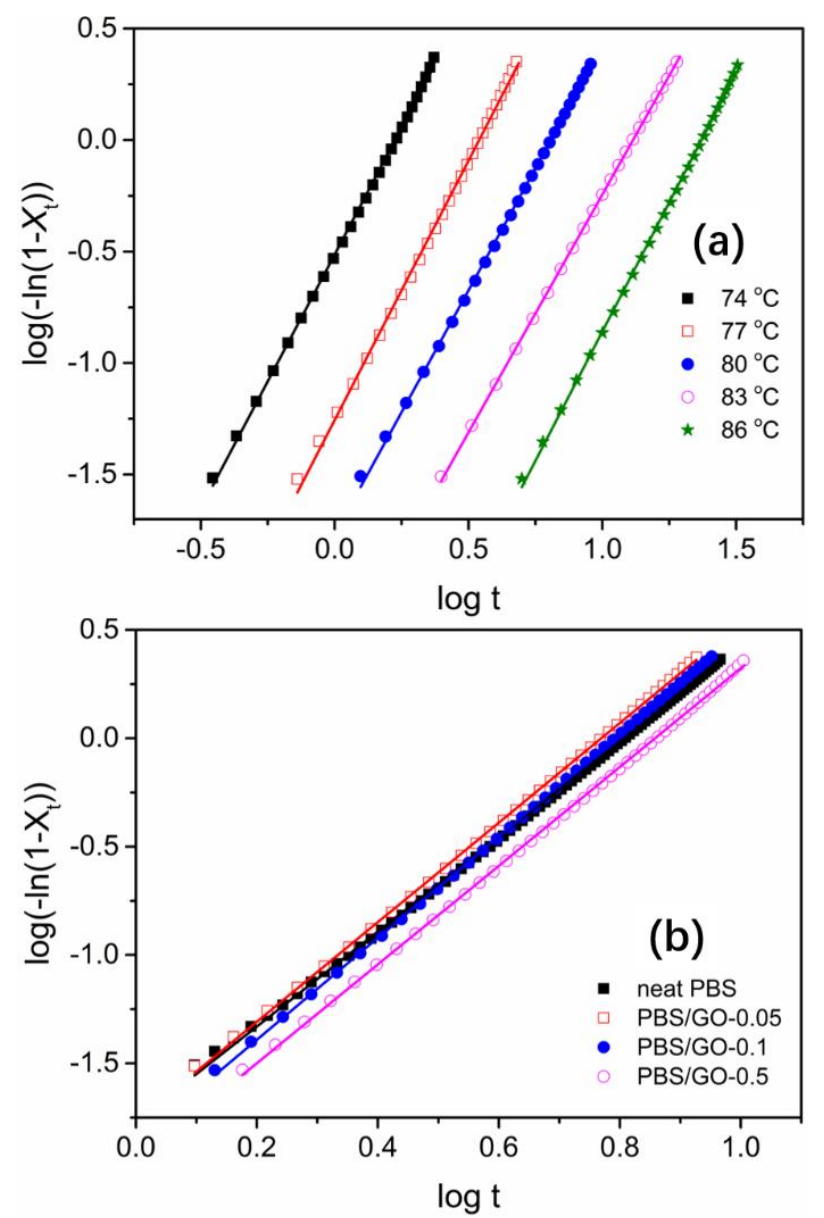

Figure 5. (a) Avrami plots for isothermal crystallization of neat PBS at various crystallization temperatures and (b) Avrami plots for isothermal crystallization of PBS and PBS/GO nanocomposites at $80^{\circ} \mathrm{C}$. 

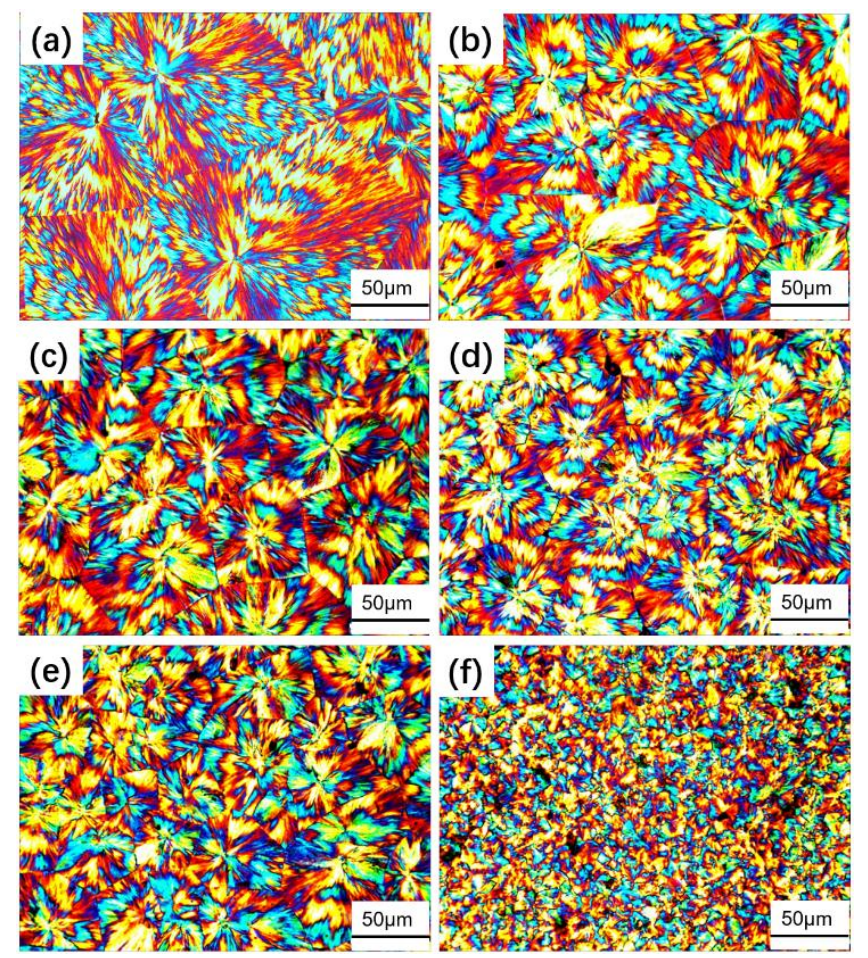

Figure 6. Spherulitic morphologies formed by isothermal crystallization at $T_{\mathrm{c}}$ of

$80{ }^{\circ} \mathrm{C}$ for neat PBS (a), PBS/GO-0.05 (b), PBS/GO-0.1 (c), PBS/GO-0.3 (d), PBS/GO-0.5 (d), and PBS/GO-1.0 (f). 


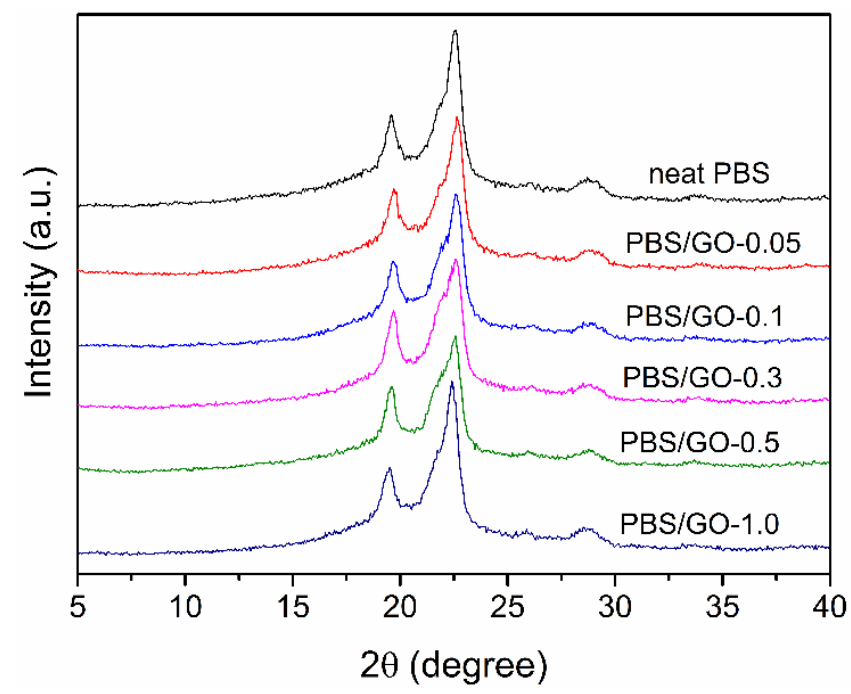

Figure 7. XRD patterns of neat PBS and PBS/GO nanocomposites. 


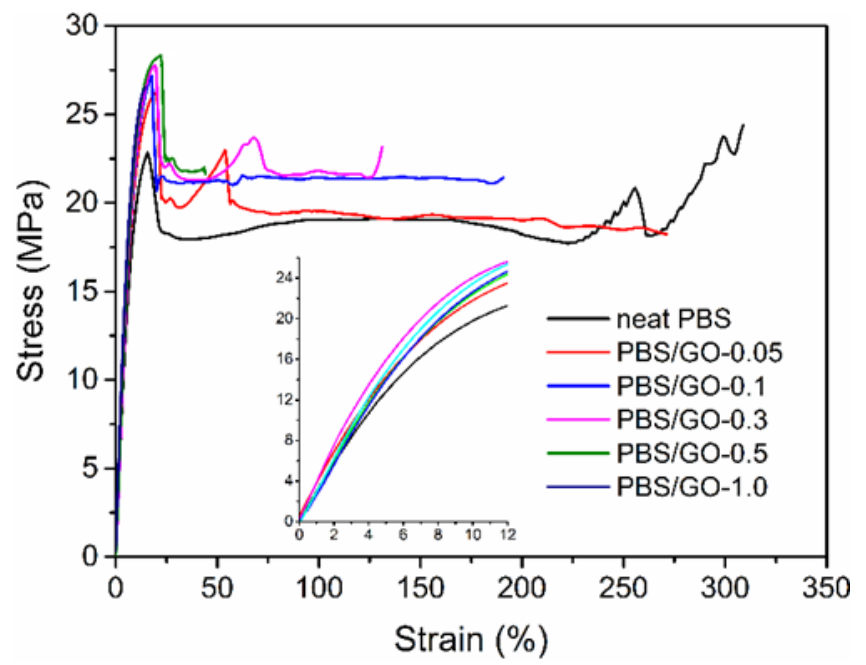

Figure 8. Stress-strain curves of neat PBS and PBS/GO nanocomposites. 


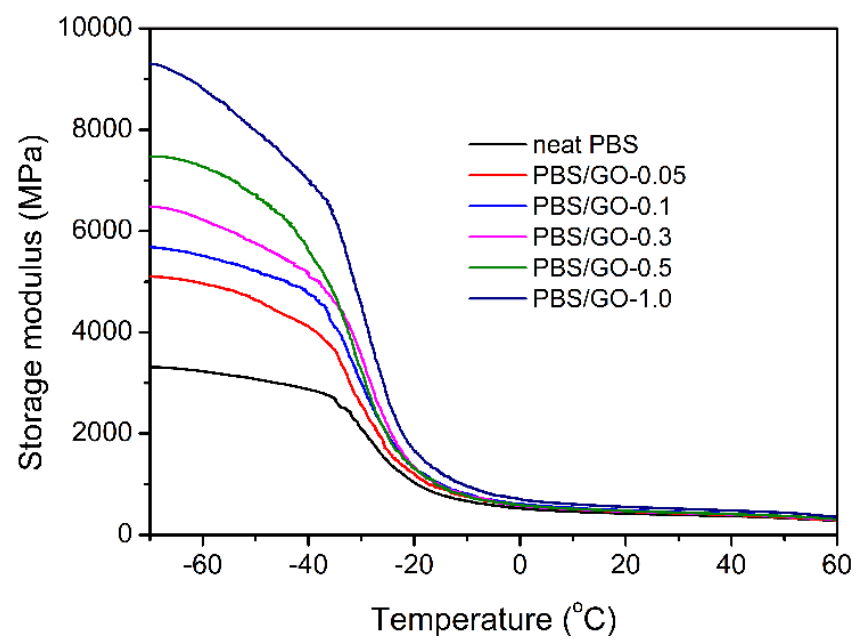

Figure 9. Storage modulus as a function of temperature for neat PBS and PBS/GO nanocomposites 